\title{
Review of: "Developing an Antiviral Peptides Predictor with Generative Adversarial Network Data Augmentation"
}

\author{
MADHURANANDA Pahar ${ }^{1}$ \\ 1 University of Stellenbosch
}

Potential competing interests: The author(s) declared that no potential competing interests exist.

This is a good well prepared research article, however needs significant improvements,

My advises to improve this paper are:

1. Use the abbreviation GAN in the abstract as it is mentioned elsewhere in the paper.

2. Please discuss more relevant studies in the Introduction. Please add them into the reference.

3. The data used here in big, please use a visual representation for your readers.

4. The paper focuses on GAN and the neural network. So, it is highly recommended that the authors explain these two methods in the paper.

5. Results need to be explained in detail with tables and a separate section 'discussion' needs to be added to highlight the findings and contributions of this paper. 